\title{
BAKER FUNCTIONS FOR COMPACT RIEMANN SURFACES
}

\author{
R. J. SCHILLING
}

\begin{abstract}
This article contains a proof of an important theorem in soliton mathematics. The theorem, stated roughly in [4], contains necessary conditions for the existence of a vector function$$
\psi(t, p)=\left(\psi_{1}(t, p), \ldots, \psi_{l}(t, p)\right), \quad t \in \mathbf{C}^{g}, \quad p \in R,
$$

with prescribed poles and $l$ essential singularities an a compact Riemann surface $R$ of genus $g . \psi$ is called a Baker function in honor of the 1928 article [1] of H. F. Baker. This report clarifies Krichever's description of $\psi$ for $l>1$ essential singularities. The divisors $\delta_{\alpha}$ in (1) below are the key to the $l>1$ construction. Krichever's $(l>1)$ construction is not easy to deal with in practical problems. E. Previato [5] noted this and applied our characterization of the $\delta_{\alpha}$ to construct the finite gap solutions to the nonlinear Schroedinger equation.
\end{abstract}

1. Baker functions. Let there be given a compact Riemann surface $R$ of genus $g$ and fix $l(\geq 1)$ distinct points, $\infty=\infty_{1}+\cdots+\infty_{l}$, of $R$. Let $\kappa_{\alpha}^{-1}$ be a local parameter vanishing at $\infty_{\alpha}$ and let $\Theta_{\alpha}$ be a polynomial in $\kappa_{\alpha}$. Let $\Delta$ be a positive nonspecial divisor on $R$. We define a complex linear space $\Lambda=\Lambda\left(\Delta, \infty, \Theta_{\alpha}\right)$ by the following properties. A function $\psi$ belongs to $\Lambda$ if

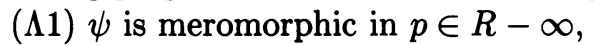

$(\Lambda 2)(\psi)+\Delta \geq 0$, i.e., any pole of $\psi$ lies in $\Delta$,

$(\Lambda 3)$ near $\infty_{\alpha}, \psi(p) e^{-\Theta_{\alpha}\left(\kappa_{\alpha}(p)\right)}$ is holomorphic.

The purpose of this paper is to compute $\operatorname{dim}_{C}(\Lambda)$. The idea is this. If the $\Theta_{\alpha}$ are "small", in a sense to be made precise, then the zero divisor $\tilde{\Delta}$ of any function in $\Lambda$ would be "close" to $\Delta$. Since the nonspecial divisors are an open set, our choice of $\Delta$ would imply that $\tilde{\Delta}$ is a nonspecial divisor of degree equal to $\operatorname{deg}(\Delta)$. This, we will see in the proof of Theorem 3 below, implies

$$
\operatorname{dim}_{\mathbf{C}}(\Lambda)=\operatorname{deg}(\Lambda)-g+1
$$

The reader may consult [2] for a current article with background on these matters.

Let us introduce a vector $t=\left(t_{0}, t_{1}, \ldots\right)$ of complex "time" parameters. Let there be given polynomials $\Theta_{\alpha}=\Theta_{\alpha}\left(t, \kappa_{\alpha}\right)$ with coefficients analytic in $t$ and $\Theta_{\alpha}\left(0, \kappa_{\alpha}\right)=0$. Let $\Delta$ be a positive nonspecial divisor of dégree $g+l-1$ such

Received by the editors January 25, 1985 and, in revised form, November 4, 1985.

1980 Mathematics Subject Classification (1985 Revision). Primary 58F07; Secondary 58F19, 14 H40.

Supported in part by NSF (Fellowship) Grant No. MCS 8211308, NSF Grant No. MCS8102748, and Department of the Army DAAG 29-82-K-0068.

I want to give special thanks to my advisor H. Flaschka, who explained the algebraic geometry underlying Baker functions, and to the referee for an important contribution to the proof of the main result. 
that $L(\Delta-\infty)=\{0\}$. The linear space $L(\Delta)$ contains a basis $f_{1}, \ldots, f_{l}$ such that $f_{\alpha}\left(\infty_{\beta}\right)=\delta_{\alpha \beta}$. Each divisor

$$
\delta_{\alpha} \stackrel{\text { def }}{=} \Delta+\left(f_{\alpha}\right)-\infty+\infty_{\alpha}=\left(\Delta-\left(f_{\alpha}\right)-\infty\right)+\left(\left(f_{\alpha}\right)_{0}-\left(\infty-\infty_{\alpha}\right)\right)
$$

$(\alpha=1, \ldots, l)$ is nonspecial and of degree $g$. The nonspeciality of $\delta_{\alpha}$ is not obvious. To see this let $f \in L\left(\delta_{\alpha}\right)$. The product $f f_{\alpha}$ has poles in $\Delta$ and zeros at each point of $\infty-\infty_{\alpha}$. Therefore, $f f_{\alpha}=$ (constant) $f_{\alpha}$ and $f=$ constant. This proves $L\left(\delta_{\alpha}\right)=\mathbf{C}$. It follows then that $\delta_{\alpha}$ is nonspecial. The following theorem is a generalization of a construction apparently due to Clebsch and Gordan. See [2]. The notations of the proof follow $[3]$.

2. Proposition. Let $\left(R, \infty, g, \kappa_{\alpha}, \Theta_{\alpha}\right)$ as above. Let $\delta$ be a nonspecial divisor of degree $g$ and supported in $R-\infty$. Then there exists precisely one function $\chi=\chi_{\delta}$ with these properties:

(2.1) $\chi$ is single valued and meromorphic in $p \in R-\infty, \chi$ is analytic in $t,|t|$ sufficiently small,

(2.2) any pole of $\chi$ lies in $\delta$,

(2.3) at $\infty_{\alpha}, \chi(t, p) e^{-\Theta_{\alpha}\left(t, \kappa_{\alpha}(p)\right)}$ is holomorphic,

$\left(2.3^{\prime}\right)$ at $\infty_{1}, \chi(t, p) e^{-\Theta_{1}\left(t, \kappa_{1}(p)\right)}=1+O\left(\kappa_{1}^{-1}\right)$.

PROOF. Let us give a formula for a function $\chi$, define the notation in the formula, verify that $\chi$ satisfies $(2.1)-\left(2.3^{\prime}\right)$, and then prove that any function satisfying (2.1) $-(2.3)$ is a scalar times $\chi$. Let

$$
\chi(t, p)=C(t) f_{t}(p) f_{0}^{-1}(p) \exp \left(\sum_{\alpha=1}^{l} \int_{p_{0}}^{p} \omega_{\alpha}(t)\right)
$$

where

$$
\begin{aligned}
f_{t}(p) & =\Theta(\Phi(p)-\Phi(\delta)-K-B(\omega(t)) / 2 \pi i) \\
C^{-1}(t) & =e^{V_{1}(t)} f_{t}\left(\infty_{1}\right) f_{0}^{-1}\left(\infty_{1}\right) \exp \sum_{\alpha=2}^{l} \int_{p_{0}}^{\infty_{1}} \omega_{\alpha}(t) .
\end{aligned}
$$

We will show that $C(t)$ gives the normalization $\left(2.3^{\prime}\right)$ at $\infty_{1}$ and $f_{t}\left(\infty_{1}\right)$ is not zero for $|t|$ small.

Notation. The function $p \rightarrow \Phi(p)$ is the Abel map of $R$ into the Jacobian variety $J$ of $R$. To construct $\Phi$ and $J$ let there be given a canonical homology basis $\alpha=\left(\alpha_{1}, \ldots, \alpha_{g}\right), \beta=\left(\beta_{1}, \ldots, \beta_{g}\right)$, with intersection matrix $\alpha_{i} \circ \alpha_{j}=\beta_{i} \circ \beta_{j}=0$ and $\alpha_{i} \circ \beta_{j}=\delta_{i j}$. Let $\phi=\left(\phi_{1}, \ldots, \phi_{g}\right)^{T}$ be the basis of holomorphic differentials with $\int_{\alpha_{i}} \phi_{j}=\delta_{i j}$. The $2 g$ columns of the matrices $I=\int_{\alpha} \phi$ and $B=\int_{\beta} \phi$ of $\alpha$ and $\beta$ periods of $\phi$ determine a lattice $L=\left\{m+B n \mid m, n \in Z^{g}\right\}$ over the integers $Z$. The Jacobian variety of $R$ is the compact, commutative, $g$-dimensional complex Lie group $J=\mathbf{C}^{g} / L$. Choose a point $p_{0} \in R-\infty$. The Abel map is defined by $\Phi(p)=\int_{p_{0}}^{p} \phi$, and $\Phi$ is extended to divisors by $\Phi\left(p_{1}+\cdots+p_{m}\right)=\sum_{j=1}^{m} \int_{p_{0}}^{p_{j}} \phi$.

The matrix $B$ of $\beta$-periods of $\phi$ is symmetric with a positive definite imaginary part. This implies that the theta function

$$
\Theta(z)=\sum_{n \in Z^{\boldsymbol{\theta}}} \exp (2 \pi i\langle z, n\rangle+\pi i\langle B n, n\rangle)
$$


is an entire function of $z \in \mathbf{C}^{g}$. One can verify by direct substitution that if $B_{j}$ is column $j$ of $B$ and $m \in Z^{g}$ then $\Theta(z+m)=\Theta(z)$ and $\Theta\left(z+B_{j}\right)=$ $\exp \left(-2 \pi i z_{j}-\pi i B_{j j}\right) \Theta(z)$.

For any $e \in \mathbf{C}^{g}$ the Riemann theta function $p \rightarrow \Theta(\Phi(p)-e)$ is either identically zero or it has precisely $g$ zeros. If $p \rightarrow \Theta(\Phi(p)-e)$ is not identically zero and if $p_{1}+\cdots+p_{g}$ is its zero divisor then $p_{1}+\cdots+p_{g}$ is nonspecial and

$$
\Phi\left(p_{1}+\cdots+p_{g}\right)+K=e
$$

$(\bmod L)$ where $K=-\sum_{j=1}^{g} \int_{\alpha_{j}} \Phi \cdot \phi_{j}+\frac{1}{2}\left(B_{11}, \ldots, B_{g g}\right) . K$ is the vector of Riemann constants.

There exists a unique abelian differential $\omega_{\alpha}(t)$ of the second kind such that (i) $\omega_{\alpha}(t)$ has zero $\alpha$-periods, (ii) the principal part of $\omega_{\alpha}(t)$ at $\infty_{\alpha}$ is $d \Theta_{\alpha}\left(t, k_{\alpha}\right)$ and (iii) $\infty_{\alpha}$ is the only pole of $\omega_{\alpha}(t)$. The $\omega_{\alpha}(t)$ vanish at $t=0$ because $\Theta\left(0, \kappa_{\alpha}\right)=0$ and the only abelian differential of the first kind with zero $\alpha$-periods is identically zero. Let $\omega(t)=\sum_{\alpha=1}^{l} \omega_{\alpha}(t)$ and let $B(\omega(t))=\int_{\beta} \omega(t)$ be the vector of $\beta$-periods of $\omega$.

Consider the function $p \rightarrow \int_{p_{0}}^{p} \omega_{1}(t)$ restricted to a small neighborhood of $\infty_{1}$ and fix a path of integration. This function has the Laurent series expansion $\Theta_{1}\left(t, \kappa_{1}\right)+$ $V_{1}(t)+O\left(\kappa_{1}^{-1}\right)$ for some scalar $t \rightarrow V_{1}(t)$. For any path of integration,

$$
\exp \int_{p_{0}}^{p} \omega_{1}(t)=e^{\Theta_{1}\left(t, \kappa_{1}(p)\right)}\left(1+O\left(\kappa_{1}^{-1}(p)\right)\right) \exp \left(V_{1}(t)+j \cdot B\left(\omega_{1}(t)\right)\right)
$$

for some integral combination $j \cdot B\left(\omega_{1}(t)\right)$ of $\beta$-periods of $\omega_{1}(t)$.

The PROPERTIES OF $\chi$. The argument of $\Theta$ in $f_{t}$ depends analytically on $t$ and it converges to that of $f_{0}$ as $t \rightarrow 0$. The zero divisor of $f_{0}$ is $\delta$. The zero divisor $\delta(t)$ of $f_{t}$ depends analytically on $t$ and it moves to $\delta$ as $t \rightarrow 0$. The nonspecial divisors are open in the space of divisors; hence, $\delta(t)$ will be nonspecial for $|t|$ sufficiently small. Since $\infty_{1} \notin \delta$ and because $\infty_{1}$ is not a pole of the $\omega_{\alpha}(t)(\alpha \geq 2)$, the scalar $C(t)$ is finite and nonzero. The normalization $\left(2.3^{\prime}\right)$ of $\chi$ follows from the definition of $C(t)$ and $V_{1}(t)$. Let us show that $\chi$ is single valued.

The dissected form of $R$ is a $4 g$ sided polygon $\prod_{j=1}^{g} \alpha_{j} \beta_{j} \alpha_{j}^{-1} \beta_{j}^{-1}$ with the edge $\alpha_{j}\left(\beta_{j}\right)$ identified with the edge of $\alpha_{j}^{-1}\left(\beta_{j}^{-1}\right)$. Let $q \in \alpha_{j}$ be identified with $\bar{q} \in \alpha_{j}^{-1}$ and let $p \in \beta_{j}$ be identified with $\bar{p} \in \beta_{j}^{-1}$. The identities ([3, p. 285, formulas (1.5)] with $\varepsilon=\varepsilon^{\prime}=0$ )

$$
\begin{aligned}
& f_{t}(\bar{p})=f_{t}(p) \\
& f_{t}(\bar{q})=f_{t}(q) \exp \left(-\pi i B_{j j}-2 \pi i\left(\Phi_{j}(q)-\Phi_{j}(\delta)-K_{j}\right)\right) e^{-B_{j}(\omega(t))}, \\
& \int_{\bar{q}}^{q} \omega=-\sum_{\alpha=1}^{l} B_{j}\left(\omega_{\alpha}(t)\right)=-B_{j}(\omega(t)), \quad \int_{\bar{p}}^{p} \omega=\sum_{\alpha=1}^{l} \int_{\alpha_{j}} \omega_{\alpha}(t)=0,
\end{aligned}
$$

imply that $\chi$ is single valued.

UNIQUENESS OF $\chi$. Suppose $\hat{\chi}(t, p)$ is another function with properties (2.1)(2.3). The quotient $\sigma=\hat{\chi}(t, p) \chi^{-1}(t, p)$ is a meromorphic function of $p$. The divisor of poles of $\sigma$ is contained in the (zero) divisor of $f_{t}$; it is nonspecial for $|t|$ small. The only function with poles in a nonspecial divisor of degree $g$ is constant. Thus $\sigma=\sigma(t)$. 
The following theorem reducesd to (2.2) when $l=1$. It is the main result of this paper. There are two keys to its proof: the divisors $\delta_{\alpha}$ defined in (2.1) and a nice generalization of the uniqueness argument in (2.2) due to the referee.

3. THEOREM. Let $R$ be a Riemann surface of genus $g$. Fix $l$ points $\infty=$ $\infty_{1}+\cdots+\infty_{l}$. Let $\Delta$ be a positive nonspecial divisor of degree $g+l-1$ such that $L(\Delta-\infty)=\{0\}$. Choose a local parameter $z$ for a neighborhood of $\infty_{\alpha}$ such that $z_{\alpha}\left(\infty_{\alpha}\right)=0$ and let $\kappa_{\alpha}=z_{\alpha}^{-1}$. Choose polynomials $\Theta_{\alpha}=\Theta_{\alpha}\left(t, \kappa_{\alpha}\right)$ in $\kappa_{\alpha}$ and analytic in $t$ such that $\Theta_{\alpha}\left(0, \kappa_{\alpha}\right)=0$. The set $\Lambda=\Lambda\left(\Delta, \infty, \Theta_{\alpha}\right)$ of functions $\chi(t, p)$ such that

(3.1) $\chi$ is meromorphic in $R-\infty$ and analytic in $t,|t|$ small,

(3.2) any pole of $\chi$ lies in $\Delta$,

(3.3) near $\infty_{\alpha}, \chi(t, p) e^{-\Theta_{\alpha}\left(t, \kappa_{\alpha}(p)\right)}$ is holomorphic,

is a complex linear space of dimension l. Moreover, there is a basis $\psi_{\alpha}$ for $\Lambda$ such that, near $\infty_{\beta}$,

$$
\left(3.3^{\prime}\right) \psi_{\alpha}(t, p) e^{-\Theta_{\beta}\left(t, \kappa_{\beta}(p)\right)}=\delta_{\alpha \beta}+O\left(\kappa_{\beta}^{-1}\right) .
$$

ProOF. We apply Proposition (2.2) with $\delta=\delta_{\alpha}$ in (1) to obtain a function $\chi_{\alpha}$ with divisor of poles $\delta_{\alpha}$ and normalized at $\infty_{\alpha}$. The function

$$
\psi_{\alpha}(t, p) \stackrel{\text { def }}{=} f_{\alpha}(p) \chi_{\alpha}(t, p)
$$

belongs to $\Lambda$ and it satisfies $\left(3.3^{\prime}\right)$. It is clear that the $\psi_{\alpha}(\alpha=1, \ldots, l)$ are linearly independent and therefore $\operatorname{dim}(\Lambda) \geq l$. We have to show that $\operatorname{dim}(\Lambda)$ is not greater than $l$. Let $\psi$ be any element of $\Lambda$. There exists a positive divisor $\Delta_{\psi}(t)$ such that $(\psi(t, \cdot))=\Delta_{\psi}(t)-\Delta$. We have $\Delta_{\psi}(t)=(\psi(t, \cdot))_{0}$ unless a zero of $\psi$ cancels a point of $\Delta$ at some instant in time. In the latter case we have $\Lambda_{\psi}(t)=(\psi(t, \cdot))_{0}+$ (cancelled points of $\Delta$ ). We have

$$
\begin{aligned}
\left(\psi_{\alpha}\right) & =\left(f_{\alpha}\right)+\left(\chi_{\alpha}\right)=\left(f_{\alpha}\right)+\left(\chi_{\alpha}\right)_{0}-\delta_{\alpha} \\
& =\left(f_{\alpha}\right)+\left(\chi_{\alpha}\right)_{0}-\left(\Delta+\left(f_{\alpha}\right)-\infty+\infty_{\alpha}\right) \quad(\text { by }(1)) \\
& =\left(\chi_{\alpha}\right)_{0}+\left(\infty-\infty_{\alpha}\right)-\Delta, \\
\left(\psi / \psi_{1}\right) & =(\psi)-\left(\psi_{1}\right)=\Delta_{\psi}(t)-\left(\left(\chi_{1}\right)_{0}+\left(\infty-\infty_{\alpha}\right)\right) \quad \text { and } \\
\left(\psi_{\alpha} / \psi_{1}\right) & =\left(\psi_{\alpha}\right)-\left(\psi_{1}\right)=\left(\chi_{\alpha}\right)_{0}+\infty_{1}-\left(\left(\chi_{1}\right)_{0}+\infty_{\alpha}\right) .
\end{aligned}
$$

These formulas show that any pole of the $l+1$ meromorphic functions $\psi / \psi_{1}$, $\psi_{1} / \psi_{1}=1, \psi_{2} / \psi_{1}, \ldots, \psi_{l} / \psi_{1}$ lies in $\left(\chi_{\alpha}\right)_{0}+\infty-\infty_{1}$, a nonspecial divisor of degree $g+l-1$. The $l+1$ functions are, by the Riemann-Roch theorem, linearly dependent.

Note. The referee has informed me of the following characterization of the important divisor $\delta_{\alpha}$. It is the unique integral divisor of degree $g$ such that

$$
\Phi(\Delta)-\Phi\left(\infty-\infty_{\alpha}\right)=\Phi\left(\delta_{\alpha}\right)
$$

Note. Let $\delta$ be an integral nonspecial divisor of degree $g$ supported in $R-\infty$ and let $p_{1}, \ldots, p_{l-1}$ be distinct points in $R-\infty$. By the Riemann-Roch there exists a nonconstant function $g_{j} \in L\left(\delta+p_{j}\right)$ such that $g_{j}\left(\infty_{j}\right)=0$. Since $\delta$ is nonspecial, $g_{j}$ has a pole at $p_{j}$ and it is unique up to a constant factor. Let $g_{l}=1$. The $l$ functions $g_{1}, \ldots, g_{l}$ are a basis for $L\left(\delta+p_{1}+\cdots+p_{l-1}\right)$ and the divisor

$$
\Delta \stackrel{\text { def }}{=} \delta+\left(p_{1}+\cdots+p_{l-1}\right)
$$


satisfies the hypothesis of Theorem 3 (i.e. $L(\Delta)=\{0\}$ ) if and only if the determinant of the $l \times l$ matrix $\left(g_{j}\left(\infty_{k}\right)\right)$ is nonzero. Thus we have a way of searching for divisors $\Delta$ satisfying $L(\Delta-\infty)=0$. The method is probably not practical unless $R$ is hyperelliptic because our explicit formulas for $g_{j}$ are quite unmanageable even for a trigonal $R$.

\section{REFERENCES}

1. H. F. Baker, Commutative ordinary differential operators, Proc. Roy. Soc. A 118 (1928), 584-593.

2. B. A. Dubrovin, Theta-functions and nonlinear equations, Russian Math. Surveys 36 (1981), 11-92.

3. H. Farkas and I. Kra, Riemann surfaces, Springer-Verlag, 1980.

4. I. M. Krichever, Integration of nonlinear equations by methods of algebraic geometry, Funktsional. Anal. i Prilozhen. 11 (1977), 15-31.

5. E. Previato, Hyperelliptic curves and solitons, Thesis, Harvard University, 1983.

Department of Mathematics, Louisiana State University, Baton Rouge, LOUISIANA 70803-4918 